\title{
Magnetic resonance imaging quality and volumes of brain structures from live and postmortem imaging of California sea lions with clinical signs of domoic acid toxicosis
}

\author{
Eric W. Montie ${ }^{1,2, *}$, Elizabeth Wheeler ${ }^{2}$, Nicola Pussini ${ }^{2}$, Thomas W. K. Battey ${ }^{3}$, \\ Jerome Barakos $^{4}$, Sophie Dennison ${ }^{2}$, Kathleen Colegrove ${ }^{5}$, Frances Gulland ${ }^{2}$ \\ ${ }^{1}$ College of Marine Science, University of South Florida, Florida 33701, USA \\ ${ }^{2}$ Veterinary Science Department, The Marine Mammal Center, Sausalito, California 94965, USA \\ ${ }^{3}$ Eckerd College, Galbraith Marine Science Center, St. Petersburg, Florida 33711, USA \\ ${ }^{4}$ California Pacific Medical Center, University of California, San Francisco, California 94143, USA \\ ${ }^{5}$ Zoological Pathology Program, College of Veterinary Medicine at Urbana-Champaign, Maywood, Illinois 60153, USA
}

\begin{abstract}
Our goal in this study was to compare magnetic resonance images and volumes of brain structures obtained alive versus postmortem of California sea lions Zalophus californianus exhibiting clinical signs of domoic acid (DA) toxicosis and those exhibiting normal behavior. Proton density(PD) and T2-weighted images of postmortem-intact brains, up to $48 \mathrm{~h}$ after death, provided similar quality to images acquired from live sea lions. Volumes of gray matter (GM) and white matter (WM) of the cerebral hemispheres were similar to volumes calculated from images acquired when the sea lions were alive. However, cerebrospinal fluid (CSF) volumes decreased due to leakage. Hippocampal volumes from postmortem-intact images were useful for diagnosing unilateral and bilateral atrophy, consequences of DA toxicosis. These volumes were similar to the volumes in the live sea lion studies, up to $48 \mathrm{~h}$ postmortem. Imaging formalin-fixed brains provided some information on brain structure; however, images of the hippocampus and surrounding structures were of poorer quality compared to the images acquired alive and postmortem-intact. Despite these issues, volumes of cerebral GM and WM, as well as the hippocampus, were similar to volumes calculated from images of live sea lions and sufficient to diagnose hippocampal atrophy. Thus, postmortem MRI scanning (either intact or formalin-fixed) with volumetric analysis can be used to investigate the acute, chronic and possible developmental effects of DA on the brain of California sea lions.
\end{abstract}

KEY WORDS: Domoic acid · California sea lion · Magnetic resonance imaging · MRI · Brain · Hippocampus · Marine mammal

\section{INTRODUCTION}

Magnetic resonance imaging (MRI) is emerging as a useful tool for studying the neuroanatomy of marine mammals. MRI-based atlases have been completed for numerous species, including the beluga whale Delphinapterus leucas (Marino et al. 2001a), common dolphin Delphinus delphis (Marino et al. 2001b), bottlenose dolphin Tursiops truncatus (Marino et al. 2001c), harbor porpoise Phocoena phocoena (Marino et al. 2003a), dwarf sperm whale Kogia simus (Marino et al. 2003b), spinner dolphin Stenella longirostris orientalis (Marino et al. 2004a) and killer whale Orcinus orca (Marino et al. 2004b). These atlases were created from MRI scans of brains that were removed from the skull and formalin fixed. To avoid dissection and fixation artifacts, neuroanatomy studies of the Atlantic white-sided dolphin Lagenorhynchus acutus were completed from MRI scans of fresh, postmortem brains intact within the 
skull, with the head still attached to the body (Montie et al. 2007, 2008). More recently, a neuroanatomical MRI-based atlas was completed from magnetic resonance (MR) images of a live California sea lion Zalophus californianus (Montie et al. 2009).

Regardless of how the brain is imaged (i.e. live, fresh and intact within the skull, removed and formalin fixed), MRI has its advantages over traditional histological methods. MRI allows thin virtual sections ( $2 \mathrm{~mm}$ in thickness) of the entire brain to be acquired efficiently and quickly, where gross and histological sectioning is not practical for viewing the entire brain. Thus, MRI can be used as a screening tool to identify lesions for further histological analysis. In addition, MRI coupled with image analysis can accurately determine the volumes of brain structures (a technique called volumetric neuroimaging), while traditional dissection and photography introduces error in performing quantitative measurements. Three-dimensional (3D) models of brain structures constructed from MRI scans can provide a valuable tool to examine spatial relationships among brain structures. In fact, volumetric neuroimaging and 3D modeling have illustrated their powerful utility in studying the brains of the Atlantic white-sided dolphin and California sea lion (Montie et al. 2008, 2009).

Traditionally, MRI has been used as a tool in diagnosing causes of neurological disease. In the case of marine mammals, there is a risk of neurological disorders associated with exposure to: (1) anthropogenic chemicals, such as polychlorinated biphenyls (PCBs) (Zoeller et al. 2002, Sharlin et al. 2006) and polybrominated diphenyl ethers (PBDEs) (Costa \& Giordano 2007), that affect neurodevelopment; (2) terrestrial pathogens that can cause brain lesions (Conrad et al. 2005); (3) noise pollution that may disrupt normal dive behavior and potentially cause gas-bubble lesions in the brain (Jepson et al. 2003); and (4) marine neurotoxins associated with harmful algal blooms that can cause necrosis in brain structures such as the hippocampus (Scholin et al. 2000, Silvagni et al. 2005). MRI and volumetric neuroimaging would be very useful techniques to assess the effects of these stressors on the neurological health of marine mammals.

A rising health concern for California sea lions is neurological disease associated with exposure to domoic acid (DA), a marine neurotoxin produced by diatoms belonging to the genus Pseudo-nitzschia (Scholin et al. 2000, Silvagni et al. 2005, Goldstein et al. 2008, Ramsdell \& Zabka 2008). In 1998, more than 400 sea lions were exposed to DA through contaminated prey (Scholin et al. 2000). Sea lions that died acutely and contained detectable levels of DA in blood and urine exhibited lesions in the hippocampus (Scholin et al. 2000). These lesions were characterized by neuronal necrosis in the hippocampal formation, leading to atrophy of this brain structure (Silvagni et al. 2005). Evidence also suggests that chronic exposure to sublethal levels of DA can lead to varying degrees of hippocampal atrophy, both unilateral and bilateral, in California sea lions (Goldstein et al. 2008). Now, there is concern that low levels of DA exposure in the developing fetus and neonate, as well as co-exposure to anthropogenic pollutants such as dichlorodiphenyltrichloroethane (DDTs), may cause subtle changes in the brain that may result in long-term cognitive impairment (Ramsdell \& Zabka 2008, Tiedeken \& Ramsdell 2010). Volumetric neuroimaging would be a useful method to determine subtle changes in morphology of the hippocampus due to varying DA exposure levels and scenarios.

In some cases, due to logistical and ethical concerns, it is advantageous to image the brain postmortem, either intact within the skull, with the head still attached to the body (i.e. postmortem-intact), or removed and formalin fixed (i.e. postmortem-fixed). Postmortem changes in the brain may affect MRI quality, introduce artifacts and affect volumes, making diagnosis of DA toxicosis difficult. Our goal in this study was to compare MR images and volumes of brain structures obtained from live and postmortem MRI scans of non-DA and DA intoxicated California sea lions. Specifically, the objectives were to: (1) compare MR images and brain structure volumes obtained from MRI scans completed on live sea lions, then on brains at 2, 24, and $48 \mathrm{~h}$ postmortem-intact; (2) compare MR images and brain structure volumes obtained from MRI scans completed on live sea lions versus imaging completed on brains that were postmortem-fixed; and (3) determine the usefulness of postmortem imaging in diagnosing DA toxicity.

\section{MATERIALS AND METHODS}

Source and processing of samples. California sea lions used in this study stranded live along the California coast from Marin to San Luis Obispo counties between 2007 and 2008 (Table 1). Sea lions were taken to The Marine Mammal Center, Sausalito, for clinical assessment, treatment and rehabilitation. Sex was determined based on genital morphology, while age class was based on body length, tooth size and stage of sagittal crest development (Greig et al. 2005). Blood samples were collected for serology, serum biochemistry and hematology. Domoic acid intoxication was characterized by clinical signs that included ataxia, head weaving or seizures. Symptoms also included marked lethargy and inappetance, vomiting, muscular twitching and central blindness blepharospasm (Goldstein et al. 2008). Serum, urine and feces samples were 


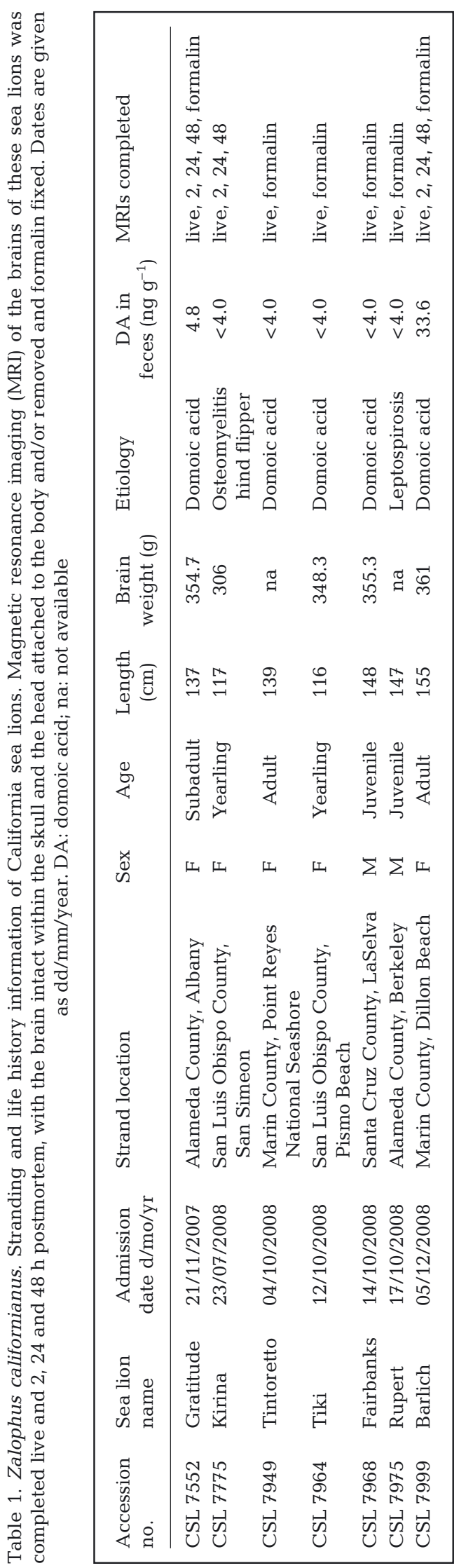

analyzed for DA by direct competitive DA ELISA (Biosense Laboratories).

Because prognosis of all sea lions in this study was poor, they were euthanized, which allowed for the comparisons of MR images and volumes of brain structures obtained and derived from live and postmortem scanning. The first data set comprised MRI scans of live sea lions performed on animals nos. CSL 7552 (named 'Gratitude'), CSL 7775 ('Kirina') and CSL 7999 ('Barlich'). The brains of these sea lions were then scanned 2, 24 and $48 \mathrm{~h}$ postmortem, with the brain intact within the skull and the head attached to the body (i.e. postmortemintact). In between postmortem-scan time points, specimens were temporarily stored at $4^{\circ} \mathrm{C}$. The second data set comprised MRI scans of live sea lions performed on CSL 7968 ('Fairbanks'), CSL 7964 ('Tiki'), CSL 7975 ('Rupert'), CSL 7949 ('Tintoretto'), CSL 7999 ('Barlich') and CSL 7552 ('Gratitude'). At necropsy, the brains of these sea lions were removed and immersed in $10 \%$ neutral buffered formalin. The elapsed time from death to immersion of brain in formalin varied between specimens (Table 2). Specimens were stored at $4^{\circ} \mathrm{C}$ until the brain was removed and fixed. At the time of MRI, the fixed brain was removed from the formalin container, dried, placed in a Ziploc bag and scanned (i.e. postmortemfixed). The elapsed time from immersion of the brain in formalin to brain scanning varied between specimens (Table 2). Following postmortem MRI, brains were sectioned for histology in the oblique plane perpendicular to the long axis of the sylvian fissure and temporal lobe. Tissues were processed routinely, embedded in paraffin, sectioned at $5 \mu \mathrm{m}$ and stained with hematoxylin and eosin (H\&E). All studies were conducted in accordance with institutional, national and international guidelines concerning the use of animals in research.

Magnetic resonance data acquisition. Radiographs of the head and chest of each individual were taken to rule out the presence of any metal that would result in image artifacts and/or injure the sea lion. Live sea lions anesthetized with isoflurane, intact specimens, and fixed brains were imaged with a 1.5-T Siemens Magnetom Symphony scanner (Siemens) equipped with

Table 2. Zalophus californianus. Time to fixation and magnetic resonance imaging (MRI) of California sea lion brains

\begin{tabular}{|lcc|}
\hline $\begin{array}{l}\text { Sea lion } \\
\text { name }\end{array}$ & $\begin{array}{c}\text { Elapsed time: } \\
\text { death to fixation }(\mathrm{h})\end{array}$ & $\begin{array}{c}\text { Elapsed time: } \\
\text { fixation to MRI (d) }\end{array}$ \\
\hline $\begin{array}{l}\text { Gratitude } \\
\text { Tintoretto }\end{array}$ & 51 & 518 \\
Tiki & 23 & 210 \\
Fairbanks & 3.5 & 199 \\
Rupert & 2 & 196 \\
Barlich & 4 & 191 \\
\hline
\end{tabular}


a CP Extremity Coil. After the localizer scan, T1weighted images in the sagittal plane were acquired using a spoiled gradient echo (FLASH) sequence with the following parameters: $\mathrm{TR}=22 \mathrm{~ms}$, TE $=10 \mathrm{~ms}$, $\mathrm{FOV}=200 \times 200 \mathrm{~mm}$, slice thickness $=1 \mathrm{~mm}$, and voxel size $=0.3 \times 0.3 \times 1 \mathrm{~mm}$. Two-dimensional proton density- (PD) and T2-weighted images in the transverse plane were acquired using a turbo spin-echo (TSE) sequence with the following parameters: TR $=3650 \mathrm{~ms}$, $\mathrm{TE}=14 / 98 \mathrm{~ms}$ for $\mathrm{PD}$ and $\mathrm{T} 2$, respectively, slice thickness $=2.5 \mathrm{~mm}, \mathrm{FOV}=150 \times 150 \mathrm{~mm}$, and voxel size $=$ $0.3 \times 0.3 \times 2.5 \mathrm{~mm}$. Additionally, 2-dimensional PDand T2-weighted images in the oblique plane (i.e. perpendicular to the long axis of the sylvian fissure and temporal lobe) were acquired using a TSE sequence with the following parameters: $\mathrm{TR}=5470 \mathrm{~ms}$, $\mathrm{TE}=$ 14/98 $\mathrm{ms}$ for PD and T2, respectively, slice thickness = $2.5 \mathrm{~mm}, \mathrm{FOV}=160 \times 160 \mathrm{~mm}$, and voxel size $=0.3 \times 0.3$ $\times 2.5 \mathrm{~mm}$. The oblique orientation was selected to optimize viewing of the hippocampus, as previously described (Goldstein et al. 2008, Montie et al. 2009).

Anatomic identification. Anatomical structures were identified using the MRI-based brain atlas of the California sea lion (Montie et al. 2009), the brain atlas of the domestic dog Canis familiaris (beagle) (DuaSharma et al. 1970) and that of the human (Nolte \& Angevine 2000). Brain structures were labeled using nomenclature adopted from the English translation of Nomina Anatomica Veterinaria (ICVGAN 2005). MR images were created using eFilm Lite 2.1.2 (Merge Healthcare) from the Digital Imaging and Communication in Medicine (DICOM) images saved during the FLASH and TSE sequences. Left and right orientation of the images followed the traditional radiology format, where the left side of the brain appears on the right side of the image.

Volume analysis of brain structures. Initial evaluation of MR images was completed at the MRI unit. Post-processing, segmentation (i.e. assigning pixels to particular structures), 3D reconstructions and volume analysis were performed using the software program AMIRA 4.1.1 (Mercury Computer Systems). The same methods as those described in the MRI-based brain atlas of a live California sea lion (Montie et al. 2009) were used to segment brain structures, create 3D reconstructions and perform volume analysis.

For live versus 2, 24 and $48 \mathrm{~h}$ postmortem-intact comparisons, 3D reconstructions and volumes of brain structures were determined for (1) whole brain, (2) cerebrospinal fluid (CSF) of the total, left and right brain, (3) CSF of the total, left and right cerebral ventricles, (4) GM and WM of the entire brain, (5) GM and WM of the total, left and right cerebral hemispheres, and (6) GM and WM of the cerebellum including the brainstem. Volumes of the left and right hippocampus and associated structures were determined for (1) lateral ventricle (ventral horn), (2) hippocampal sulcus (which included CSF of sulcus and CSF of transverse fissure of bichat), (3) hippocampus (which included the subiculum, cornu ammonis, dentate gyrus, fimbria and alveus), and (4) parahippocampal gyrus WM and GM. Except for whole brain, structural volumes were determined on two separate occasions. Segmentations of brains were completed blindly without investigator knowledge of the clinical status of the animal (i.e. DA toxicosis or non-DA etiology).

For the live versus postmortem-fixed comparisons, $3 \mathrm{D}$ reconstructions and volumes of brain structures were determined for (1) GM and WM of the total, left and right cerebral hemispheres, (2) hippocampus and (3) parahippocampal gyrus WM and GM. Structure volumes were also determined on two separate occasions. CSF and ventricle volumes were not reported because fluid in the brain would have been replaced with formalin. GM and WM of the entire brain and cerebellum for the sea lions 'Fairbanks', 'Tiki' and 'Rupert' were not reported because a portion of the cerebellum and brainstem were removed for chemical analysis.

Data analysis. For live versus 2, 24, and $48 \mathrm{~h}$ postmortem-intact comparisons, the mean volume $\left(\mathrm{cm}^{3}\right.$ or $\mathrm{mm}^{3}$ ) and SD of these structures were reported for each sea lion (i.e. 'Gratitude', 'Kirina' and 'Barlich') at each imaging time point (i.e. live and 2, 24 and $48 \mathrm{~h}$ postmortem). The percentage of cerebral hemisphere occupied by the left or right hippocampus was calculated by dividing the structure volume (i.e. from native T2-weighted images) by the sum of the left or right cerebral WM and GM volumes (i.e. from processed PD-weighted images) multiplied by 100 . For each sea lion at each postmortem-intact time point, the percentage of the live volume for each structure was calculated by dividing the postmortem volume by the live volume multiplied by 100 . The average percentage (\%) and SD of the live volume of the 3 sea lions were reported for each structure. Data analysis for the live versus postmortem-fixed comparisons followed a similar approach as postmortem-intact analysis. Statistical analysis was not completed because of low sample sizes (i.e. $\mathrm{n}=3$ for live versus postmortem-intact comparisons; $\mathrm{n}=6$ for live versus postmortem-fixed comparisons).

\section{RESULTS AND DISCUSSION}

\section{MR imaging: live versus 2,24 and $48 \mathrm{~h}$ postmortem-intact}

The FLASH sequences of sea lions 'Gratitude', 'Kirina' and 'Barlich' revealed changes in image qual- 
ity of the brain as postmortem time increased, which indicated that this acquisition protocol was not useful as a postmortem diagnostic tool. The images that were acquired while the sea lions were alive illustrated a distinct contrast between GM and WM of the brain (Fig. S1A in the supplement available at www.int-res.com/articles/suppl/d091p243_supp.pdf). As postmortem time increased, the contrast between GM and WM was lost. The images acquired alive and postmortem-intact revealed a high-intensity signal in the posterior pituitary gland, which was present in all sea lions (Fig. S1). This finding is normal and commonly seen on T1-weighted images (Colombo et al. 1987). Evidence suggests that the high-intensity signal originates from lipids or lipid-like material, which may be linked to hormone production (Colombo et al. 1987).

Because of the lack of contrast between the GM and WM in the postmortem-intact images, the FLASH sequence was not a reliable MRI sequence for postmortem evaluation of the brain. Thus, our focus shifted to using PD- and T2-weighted images acquired during the TSE sequences. PD-weighted transverse images acquired live and postmortem-intact revealed excellent contrast between GM and WM of the cerebral hemispheres and cerebellum (Fig. S2 in the supplement). In the PD- and T2-weighted transverse images of the live sea lions, the mesencephalic aqueduct was identified but characterized by a signal void (Figs. S2 \& S3 in the supplement). The lack of CSF signal in the aqueduct represents a flow artifact secondary to pulsatile CSF flow (Feinberg \& Mark 1987, Malko et al. 1988, Lisanti et al. 2007). However, on postmortem brain images, CSF signal in the mesencephalic aqueduct and vasculature was present in the PD- and T2weighted transverse images due to lack of flow. As expected, the transverse PD-weighted images were better for visualizing WM and GM contrast (Fig. S2), while the T2-weighted images were better for identifying CSF (Fig. S3).

In the images acquired live and postmortem-intact, the structures of the hippocampal formation were best visualized in the oblique PD- and T2-weighted images, as noted in the non-DA neurologically normal California sea lion 'Kirina' (Figs. 1 \& S4 in the supplement). The cornu ammonis or hippocampus proper and the subiculum were easily visualized up to $48 \mathrm{~h}$ postmortem (Figs. 1 \& S4). The parahippocampal gyrus was easily recognized in the postmortem MR images. WM tracts of the hippocampus, the alveus and fimbria, and WM tracts of the parahippocampal gyrus were identifiable in the postmortem images; however, the boundaries between WM and GM of these structures were more visible in the live and $2 \mathrm{~h}$ postmortem images. Thus, imaging brains postmortem, up to $48 \mathrm{~h}$ after death, provides adequate images of the hippocampus and surrounding structures.

The sea lions 'Gratitude' and 'Barlich' exhibited clinical signs of DA toxicosis, and their feces contained detectable concentrations of DA (Table 1). The T2weighted images derived from scanning the brain of 'Barlich' alive revealed atrophy of the right hippocampus (i.e. cornu ammonis) with ex vacuo ventriculomegaly (i.e. enlargement of the ventral horn of the right lateral ventricle) (Fig. 2A). These images also revealed thinning of the right parahippocampal gyrus. These findings were also observed in the T2-weighted images obtained postmortem (Fig. 2B-D). However, the WM of the subiculum and parahippocampal gyrus was not as well defined and blended in with the GM. CSF surrounding the hippocampus was more prevalent in the postmortem images.

\section{Brain volumes: live versus 2,24 and $48 \mathrm{~h}$ postmortem-intact}

Volumes of all brain structures derived from imaging sea lions alive are reported in Table 3. Segmentation of the transverse T2-weighted images was used to delineate the brain surface and calculate whole brain volume for sea lions imaged alive and 2, 24 and $48 \mathrm{~h}$ postmortem-intact (Fig. S5 in the supplement). The whole brain volumes that were determined from images acquired alive were similar to the volumes obtained from images acquired postmortem-intact (Fig. S5). The volumes of total CSF and ventricles were determined from segmentations of the transverse T2-weighted images after digital removal of nearby blubber, muscle, skull and other head anatomy (Fig. S5, Table S1 in the supplement). Generally, the volumes of CSF increased at $2 \mathrm{~h}$ postmortem, then decreased at 24 and $48 \mathrm{~h}$ after death (Fig. S5, Table S1 in the supplement). This pattern is best explained by the fact that at $2 \mathrm{~h}$ postmortem the diffusion artifact caused by blood flow and CSF flow in a live animal is not present once death occurs; therefore, the volume that characterizes the high signal intensity of fluid increases, whereas at 24 and $48 \mathrm{~h}$ postmortem, the volume of CSF decreases because leakage occurs. The general pattern for ventricular volume was a decrease from live to $48 \mathrm{~h}$ postmortem, which was most probably due to CSF leakage (Fig. S5, Table S1).

The volumes of GM and WM of the brain were estimated from segmentations of the transverse PDweighted images (Fig. S5, Table S1). The GM and WM volumes of the entire brain, cerebral hemispheres and cerebellum determined from images acquired alive were, for the most part, similar to the volumes determined from images acquired postmortem 

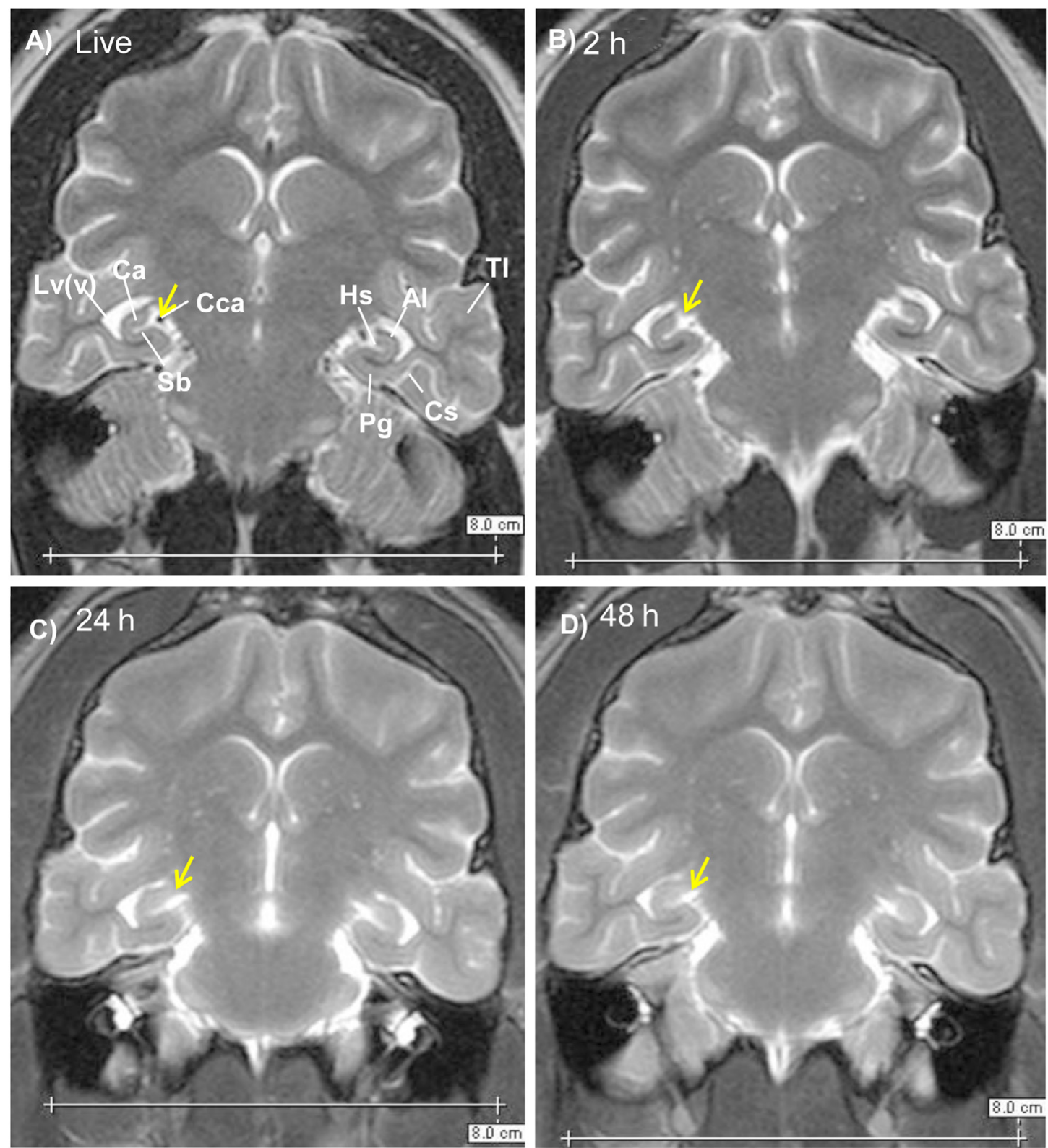

Fig. 1. Zalophus californianus. T2-weighted magnetic resonance images acquired in the oblique plane of a California sea lion ('Kirina') that was imaged (A) alive, (B) 2 h, (C) $24 \mathrm{~h}$ and (D) $48 \mathrm{~h}$ postmortem-intact. This sea lion did not show any clinical signs of domoic acid toxicosis. The diffusion artifact caused by blood flow in a live animal was not present postmortem (indicated by a yellow arrow). The T2-weighted images were still useful for examination of the hippocampus up to $48 \mathrm{~h}$ postmortem. Al: alveus; Ca: cornu ammonis; Cca: caudal cerebral artery; Cs: collateral sulcus; Hs: hippocampal sulcus; Lv(v): lateral ventricle (ventral horn); Pg: parahippocampal gyrus; Sb: subiculum; Tl: temporal lobe. Scale bar $=8.0 \mathrm{~cm}$

(Fig. S5, Table S1). Thus, imaging brains postmortemintact, up to $48 \mathrm{~h}$ after death, does not have any obvious effect on GM and WM volumes, but does affect CSF volumes.

Volumes of the left and right hippocampus and associated structures were determined by manual segmentation of oblique T2-weighted images (Fig. S6 in the supplement). The T2-weighted images were used because they were better at highlighting fluid struc- tures surrounding the hippocampus compared with the PD images (Figs. $1 \&$ S4). These fluid structures served as boundaries of the hippocampus and were defined by higher signal intensities.

For 'Kirina' (i.e. non-DA, neurologically normal female sea lion), volumes of the left and right hippocampus were approximately equal (Table 3, Fig. 3). In addition, the left and right parahippocampal gyri were approximately equal in volume (Tables 3 \& S2). 

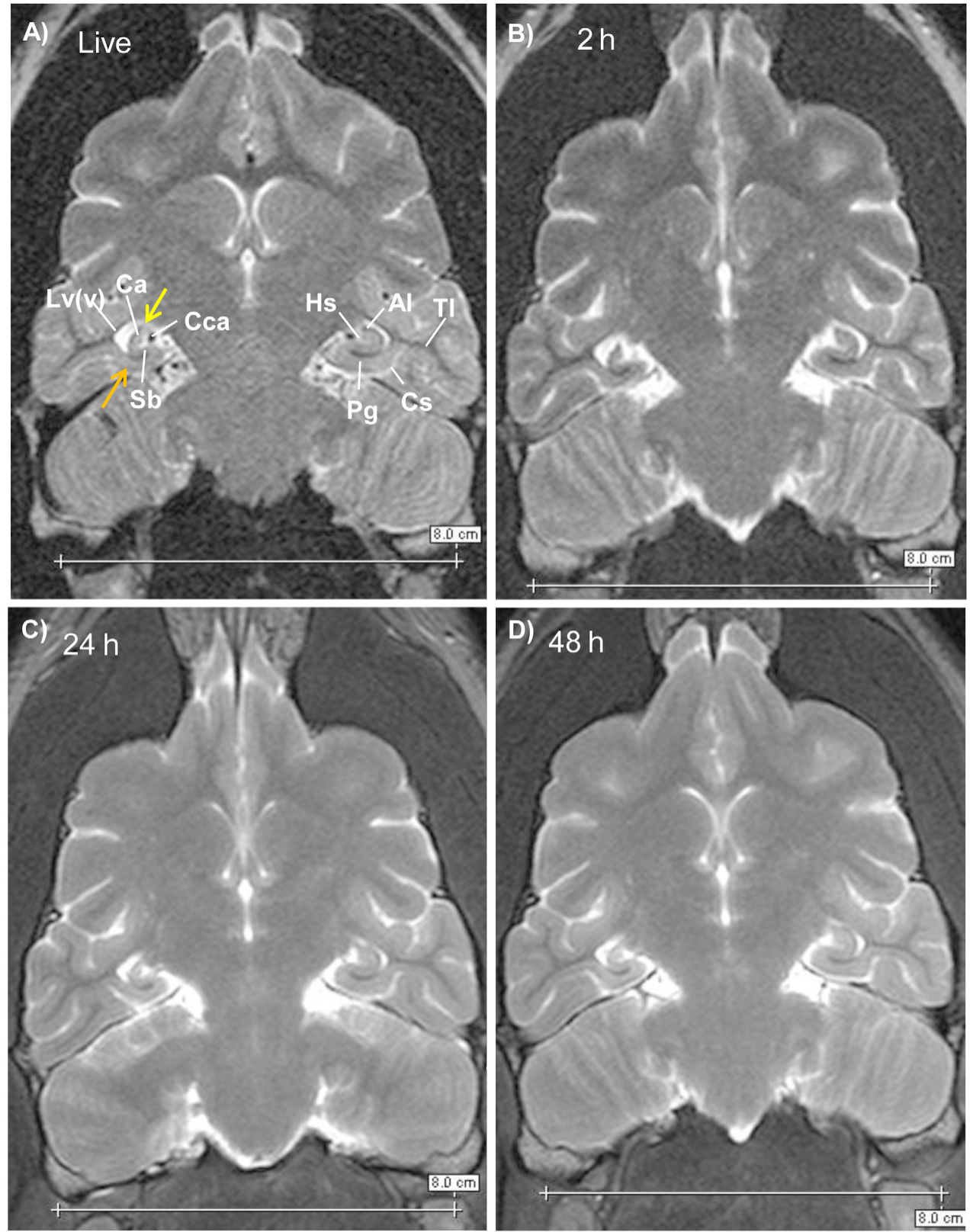

Fig. 2. Zalophus californianus. T2-weighted magnetic resonance images acquired in the oblique plane of a California sea lion ('Barlich') that contained domoic acid (DA) in feces and exhibited clinical signs of DA toxicosis. The animal was imaged (A) alive, (B) $2 \mathrm{~h}$, (C) $24 \mathrm{~h}$ and (D) $48 \mathrm{~h}$ postmortem-intact. The T2-weighted images revealed atrophy of the right hippocampus (indicated by yellow arrow) with ex vacuo ventriculomegaly. This condition presents as enlargement of the temporal horn of the right lateral ventricle. These images also revealed thinning of the right parahippocampal gyrus (indicated by orange arrow). The T2-weighted images were still useful for examination of the hippocampus up to $48 \mathrm{~h}$ postmortem. See Fig. 1 for abbreviations. Scale bar $=8.0 \mathrm{~cm}$

Both the volumes of the left and right hippocampus were $0.84 \%$ of the volumes of the left and right cerebral hemispheres (Fig. 3C-D). Postmortem-intact imaging did not have an effect on the volumes of the hippocampus or the percentages; however, the volumes of the ventral horn of the lateral ventricles decreased as postmortem imaging time increased
(Table S2 in the supplement). We attribute this finding to CSF leakage. Most importantly, volumes and percentages of the hippocampus relative to the cerebral hemispheres determined from postmortem images were similar to values calculated from images acquired alive. Histological evaluation indicated that both the left and right hippocampus were within normal limits. 


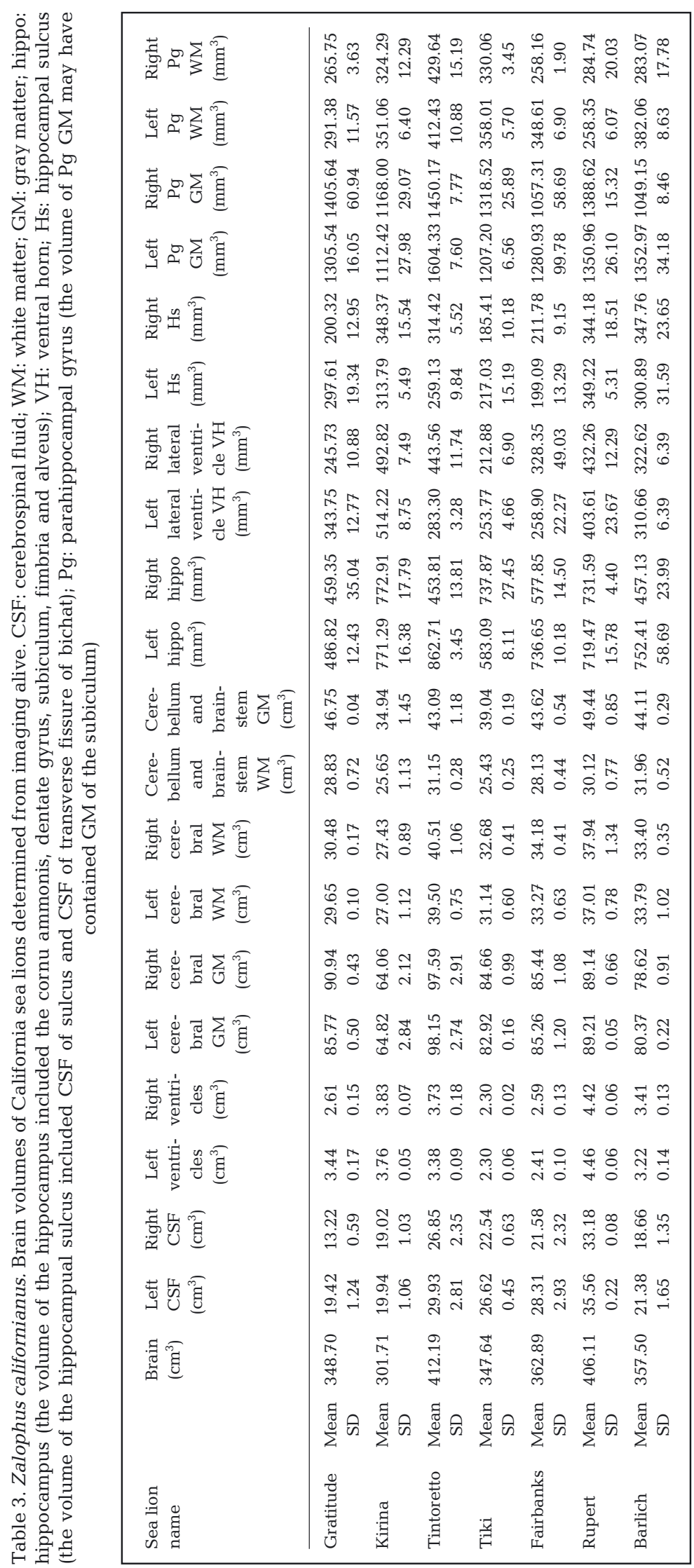

The sea lion 'Gratitude' exhibited bilateral hippocampal atrophy, which was substantiated by volumetric measurements. Even though this female California sea lion was larger than 'Kirina' (137 cm compared with $117 \mathrm{~cm}$ ) and exhibited larger total GM and WM volumes, the left and right hippocampi were smaller both in absolute and relative volumes (Table 3, Fig. 3). Volumes of the left and right hippocampi calculated from images acquired postmortem-intact were similar to the volumes determined from images acquired alive (Fig. 3). Histopathology confirmed that both the left and right hippocampi exhibited lesions consistent with DA toxicosis including atrophy, neuronal loss and gliosis. Thus, volumes of the hippocampus determined from images acquired postmortem-intact provided an accurate method in detecting bilateral hippocampal atrophy.

Sea lion 'Barlich' exhibited unilateral, right hippocampal atrophy, which was also substantiated by volumetric measurements. This adult female was $155 \mathrm{~cm}$ in length, much larger than 'Kirina', and exhibited larger total GM and WM volumes compared with the volumes observed for 'Kirina'. Yet, the right hippocampus was about half the size of that for 'Kirina' (Table 3, Fig. 3). The left hippocampus was similar in size to the left hippocampus of 'Kirina'. Volumes of the left and right hippocampi calculated from images acquired postmortemintact were similar to the volumes determined from images acquired alive (Fig. 3). Gross pathologic evaluation of 'Barlich' confirmed moderate atrophy of the right hippocampus (Fig. 4A). Neuronal and granular cell loss in the right hippocampal pyramidal cell layer and dentate gyrus and moderate gliosis were noted on histological evaluation, confirming exposure to DA (Fig. 4B). The left hippocampus was within normal limits (Fig. 4C). Thus, the volume difference between the left and right hippocampus determined from imaging correlated with the difference seen histologically. Hence, volumes of the hippocampus determined from images acquired up to $48 \mathrm{~h}$ after death provided an accurate method in detecting bilateral hippocampal atrophy.

\section{MR imaging: live versus postmortem-fixed}

The PD-weighted transverse images of the postmortem-fixed brains revealed excellent 

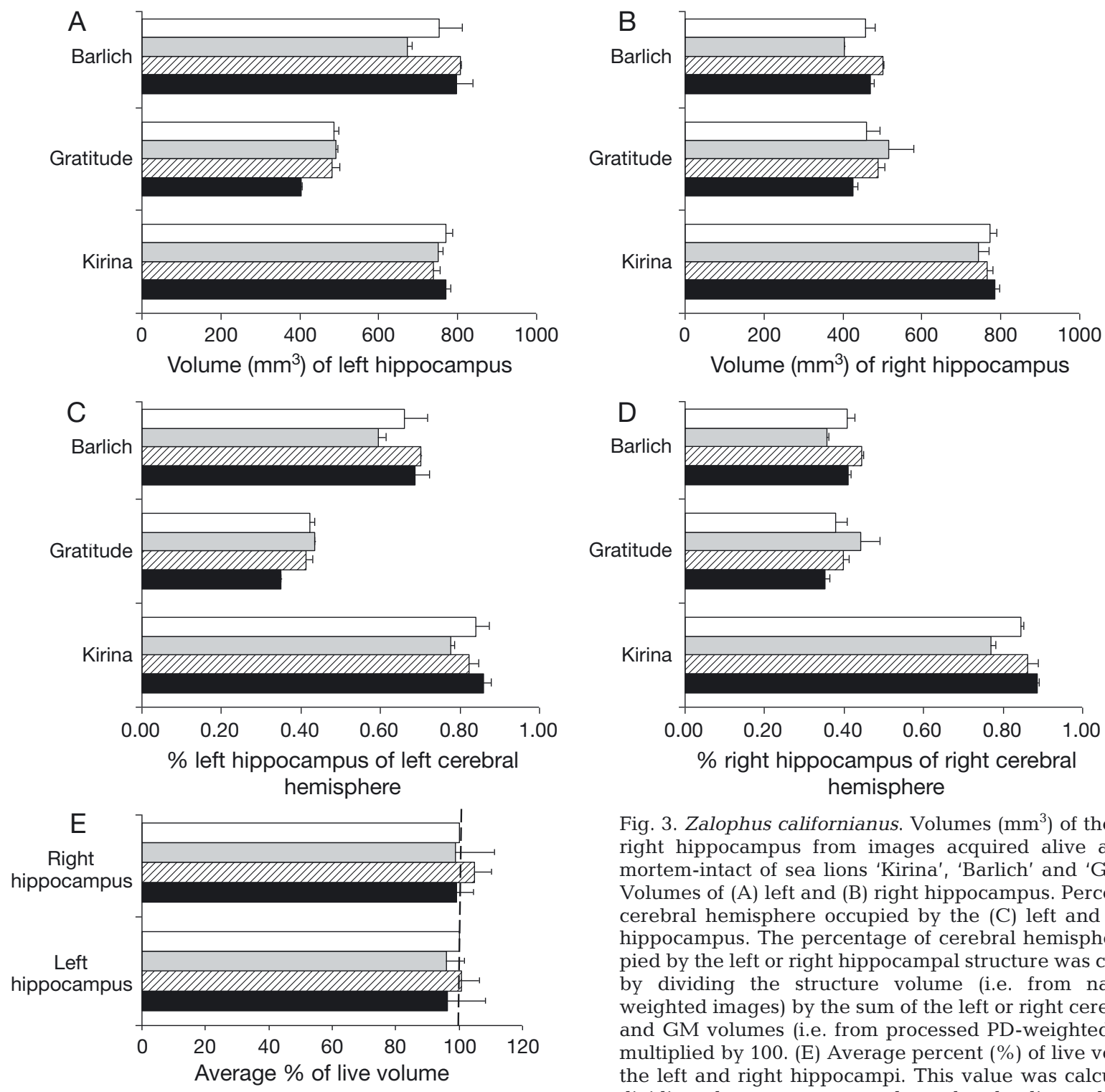

Fig. 3. Zalophus californianus. Volumes $\left(\mathrm{mm}^{3}\right)$ of the left and right hippocampus from images acquired alive and postmortem-intact of sea lions 'Kirina', 'Barlich' and 'Gratitude'. Volumes of (A) left and (B) right hippocampus. Percent of the cerebral hemisphere occupied by the (C) left and (D) right hippocampus. The percentage of cerebral hemisphere occupied by the left or right hippocampal structure was calculated by dividing the structure volume (i.e. from native T2weighted images) by the sum of the left or right cerebral WM and GM volumes (i.e. from processed PD-weighted images) multiplied by 100. (E) Average percent (\%) of live volume for the left and right hippocampi. This value was calculated by dividing the postmortem volume by the live volume and multiplying by $100 \%$ for 'Kirina', 'Barlich' and 'Gratitude'. The mean and SD of these 3 sea lions were reported. Structural volumes were determined twice, which was the source of the error bars

contrast between GM and WM, and were similar to the contrast seen in live images (Fig. S7 in the supplement). However, in some cases, the images of the formalin-fixed brains were flattened in the dorsal-ventral direction, most probably related to the weight of the brain (Fig. S7). As expected, the PD-weighted images of the postmortem-fixed brains were better for visualizing WM and GM contrast than the T2-weighted images. The elapsed time from death to fixation (up to $51 \mathrm{~h}$ ) did not affect the WM and GM contrast.

Similar to MR images acquired live, the oblique T2weighted images of postmortem-fixed brains were best

for visualizing the hippocampus (Fig. S8 in the supplement). However, the boundaries of the hippocampus were less distinct in these images compared with the boundaries in the images acquired alive (Fig. S8). The cornu ammonis or hippocampus proper and the subiculum were recognized; however, in some cases, the boundaries were blurred (Fig. S8D), while in other cases the boundaries remained distinct (Fig. S8F). These patterns were observed for other structures, including the parahippocampal gyrus, as well as WM tracts of the hippocampus, the alveus and fimbria and WM tracts of the parahippocampal gyrus. These ef- 


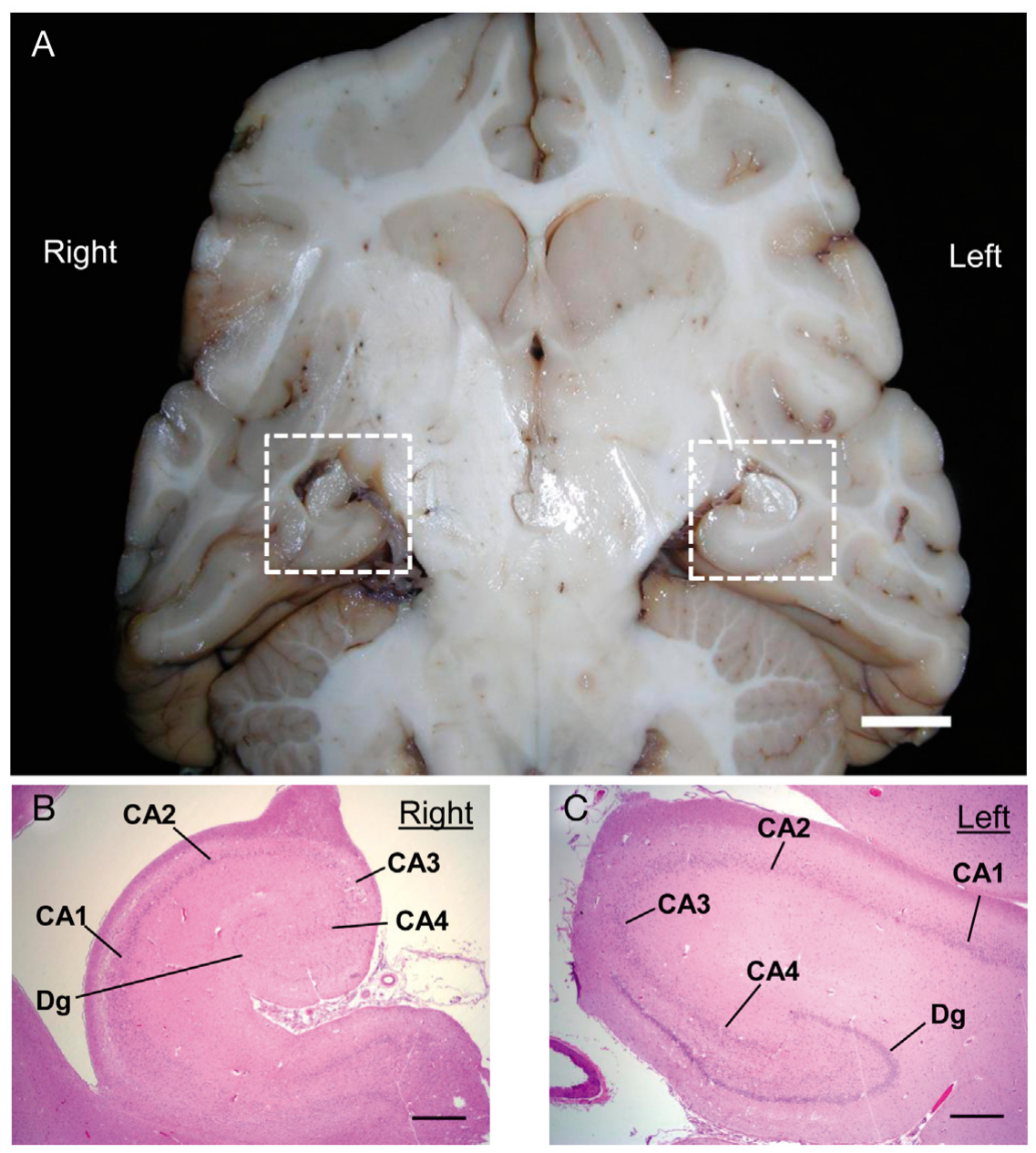

Fig. 4. Zalophus californianus. Gross and histological images of 'Barlich', a California sea lion that contained domoic acid (DA) in feces and exhibited clinical signs of DA toxicosis. (A) The gross image revealed atrophy of the right hippocampus with ex vacuo ventriculomegaly. The left hippocampus was normal. Dotted squares encompass the left and right hippocampi and parahippocampal gyrus. Scale bar $=1 \mathrm{~cm}$. (B) H\&E stain showing neuronal and granular cell loss in the right hippocampal pyramidal cell layer and dentate gyrus and moderate gliosis. (C) H\&E stain of the normal left hippocampus, which is much larger than the right hippocampus. CA: cornu ammonis; Dg: dentate gyrus. Scale bars (in B,C) $=1 \mathrm{~mm}$

fects were not associated with the elapsed time from death to fixation. Thus, imaging formalin-fixed brains provides some information on brain structure; however, the images of the hippocampus and surrounding structures were of poorer quality (i.e. with regards to contrast of WM and GM) than images acquired alive and postmortem-intact.

The sea lions 'Fairbanks', 'Tiki', 'Tintoretto', 'Barlich' and 'Gratitude' exhibited clinical signs of DA intoxication, but only 'Gratitude' and 'Barlich' contained detectable concentrations of DA in feces (Table 1). Scanning the postmortem-fixed brain, in some cases, was still useful in diagnosing hippocampal atrophy (Fig. S8). For example, the T2-weighted images derived from scanning the formalin-fixed brain of 'Barlich' revealed atrophy of the right hippocampus (i.e. cornu ammonis) (Fig. S8F). However, in other cases, scanning the formalin-fixed brain provided less than optimal images of the hippocampus, and it was difficult to detect hippocampal changes (Fig. S8B,D).

\section{Brain volumes: live versus postmortem-fixed}

Volumes of all brain structures derived from imaging sea lions alive are reported in Table 3 . The volumes of cerebral GM and WM of the postmortem-fixed brains were estimated from segmentations of the transverse PD-weighted images (Fig. 5, Table S3 in the supplement). GM and WM volumes of the cerebellum for sea lions 'Rupert', 'Tiki' and 'Fairbanks' were not determined because a portion of the cerebellum had been 
removed for chemical analysis. The GM and WM volumes of the cerebral hemispheres determined from images of formalin-fixed brains were, for the most part, similar to the volumes obtained from images acquired alive.

Volumes of the left and right hippocampi and associated structures of the postmortem-fixed brains were determined by manual segmentation of oblique T2weighted images. Imaging fixed brains did not have an obvious effect on the volumes of the hippocampi or the percentages compared with live imaging (Fig. 5). Volumetric calculations of the hippocampi from postmortem-fixed images provided an accurate method in detecting unilateral and bilateral hippocampal atrophy. The sea lion 'Gratitude' exhibited a decrease in volume of the left and right hippocampus compared with the volumes measured in 'Rupert' (a sea lion that did not show any clinical signs of DA toxicosis). Histopathology confirmed the findings for 'Gratitude', as previously discussed. On histological evaluation of 'Rupert's' brain, the hippocampi were normal. The brains of 'Barlich', 'Tintoretto' and 'Fairbanks' showed a decrease in volume of the right hippocampus, while that of 'Tiki' revealed a loss in volume of the left hippocampus (Fig. 5). Histopathology confirmed the MRI findings. Lesions consistent with DA poisoning, including hippocampal pyramidal and granular cell loss and gliosis, were only present in the right hippocampus of 'Barlich', 'Tintoretto' and 'Fairbanks' and in the left hippocampus of 'Tiki'. Thus, the volume differences between the left and right hippocampus determined from imaging formalinfixed brains correlated with the differences observed histologically.

\section{Understanding hippocampal atrophy and domoic acid toxicosis}

In the present study, volumes of the hippocampi from images acquired live, postmortem-intact, and postmortem-fixed provided an accurate method in detecting unilateral and bilateral hippocampal atrophy associated with DA toxicosis. However, it was important to express the volume of the left or right hippocampus relative to the volumes of the left or right cerebral hemispheres (i.e. percent hippocampus) because the hippocampus grows in size from pups to adults. For the female yearling ('Kirina') that did not exhibit any clinical signs of DA toxicosis, the volumes of the left and right hippocampi were $0.84 \%$ of the volumes of the left and right cerebral hemispheres. Whether this percentage can serve as a baseline normal value remains in question and will require the analysis of more normal cases. Thus, future research should focus on how the volume of the normal hippocampus varies with sex, length and age of the sea lion.

In addition, it is worthwhile to mention that using percentage hippocampus (relative to the left or right cerebral hemisphere, respectively) as a means to assess the degree of hippocampal atrophy associated with DA toxicosis does need further exploring because atrophy of other brain structures within the cerebral hemispheres may be associated with DA toxicosis. Silvagni et al. (2005) showed that sea lions with DA toxicosis exhibited necrotic neurons throughout much of the cortical and thalamic limbic system, including olfactory bulb, pyriform lobe and rostral thalamic nuclei. Voxel-based morphometry studies in humans with temporal lobe epilepsy (TLE) have shown a reduction in volume of many brain regions relative to those of healthy controls (Keller \& Roberts 2008). These structures included the cerebellum, midbrain, claustrom, globus pallidus, putamen, caudate nucleus, thalamus, occipital lobe, parietal lobe, insula, cingulate gyrus, orbital frontal lobe, dorsal frontal lobe, fornix, Heschl's gyrus, superior temporal gyrus, middle temporal gyrus, inferior temporal gyrus, fusiform gyrus, parahippocampal gyrus, entorhinal cortex, amygdala and hippocampus (Keller \& Roberts 2008). Epilepsy in California sea lions has been recently associated with chronic consequences of sublethal exposure to DA (Goldstein et al. 2008). Thus, future research should investigate how DA affects other brain structures besides the hippocampus. These investigations would be helpful in determining if percentage hippocampus is the 'gold standard' in assessing the chronic consequences of DA toxicosis in California sea lions.

It is curious that some sea lions exhibited unilateral atrophy of the hippocampus, whereas other individuals showed bilateral atrophy. Goldstein et al. (2008) also observed the occurrence of unilateral brain lesions in California sea lions exposed to sublethal levels of DA. In that study, more sea lions exhibited unilateral damage than bilateral damage, and right-sided atrophy was most common (Goldstein et al. 2008). The mechanism involved in the pathology of unilateral versus bilateral hippocampal atrophy is unclear but may be linked to seizure origin. In sea lions, it is possible that atrophy is ipsilateral to the seizure focus. Thus, if seizures occur in both hemispheres, then the animal would exhibit bilateral atrophy; if seizures originate in the right hemisphere, then the sea lion would exhibit right hippocampal atrophy. In humans with temporal lobe epilepsy, volume reduction of the hippocampus ipsilateral to the side of the seizure is the primary finding in voxel-based morphometry studies (as reviewed in Keller \& Roberts 2008). This finding supports our proposed mechanism. 


\section{CONCLUSIONS}

This investigation presents a comparison of MRI quality and volumes of brain structures obtained and derived from imaging the brain of California sea lions live, postmortem-intact, and postmortem-fixed. PD-
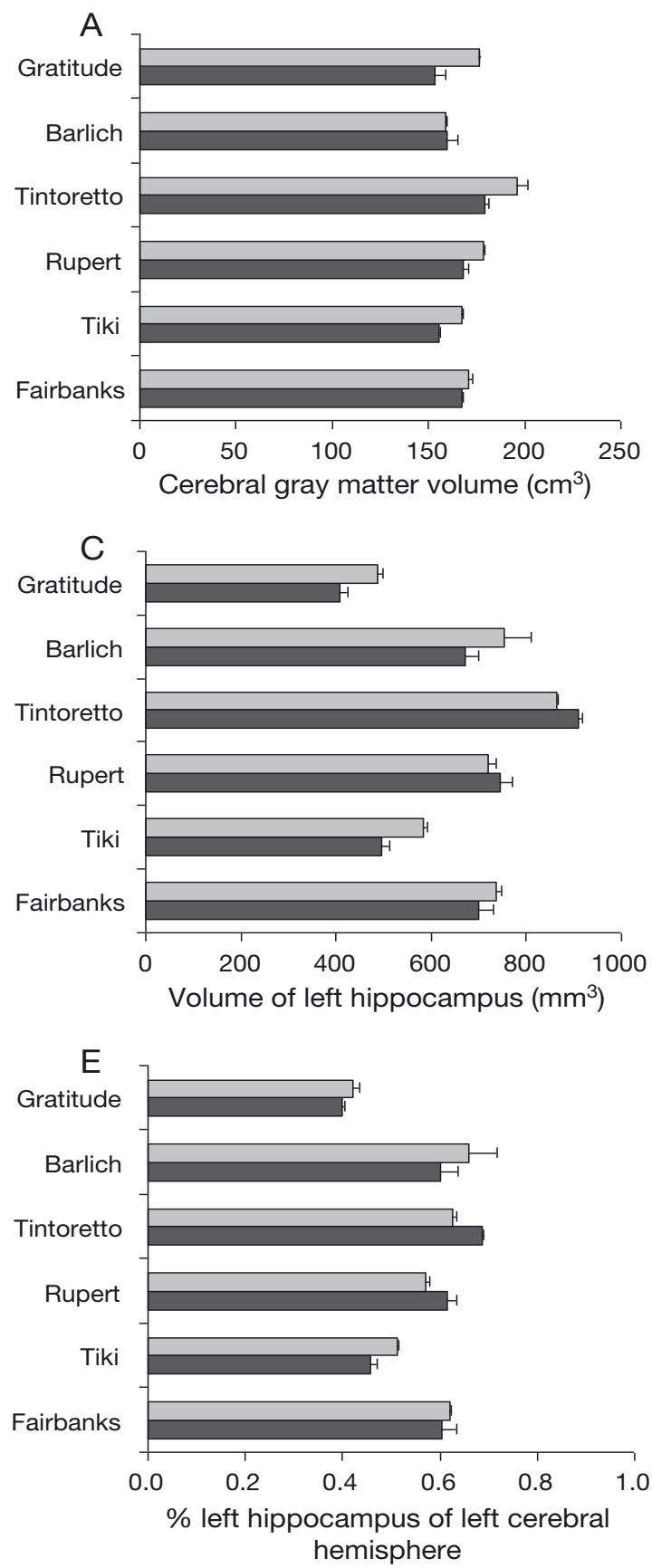

and T2-weighted images provided sufficient quality (i.e. signal to noise and contrast of WM and GM) to view the brain up to $48 \mathrm{~h}$ postmortem, which allowed the determination of volumes of brain structures that were comparable with live imaging. Volumes of the hippocampus from postmortem-intact images (up to
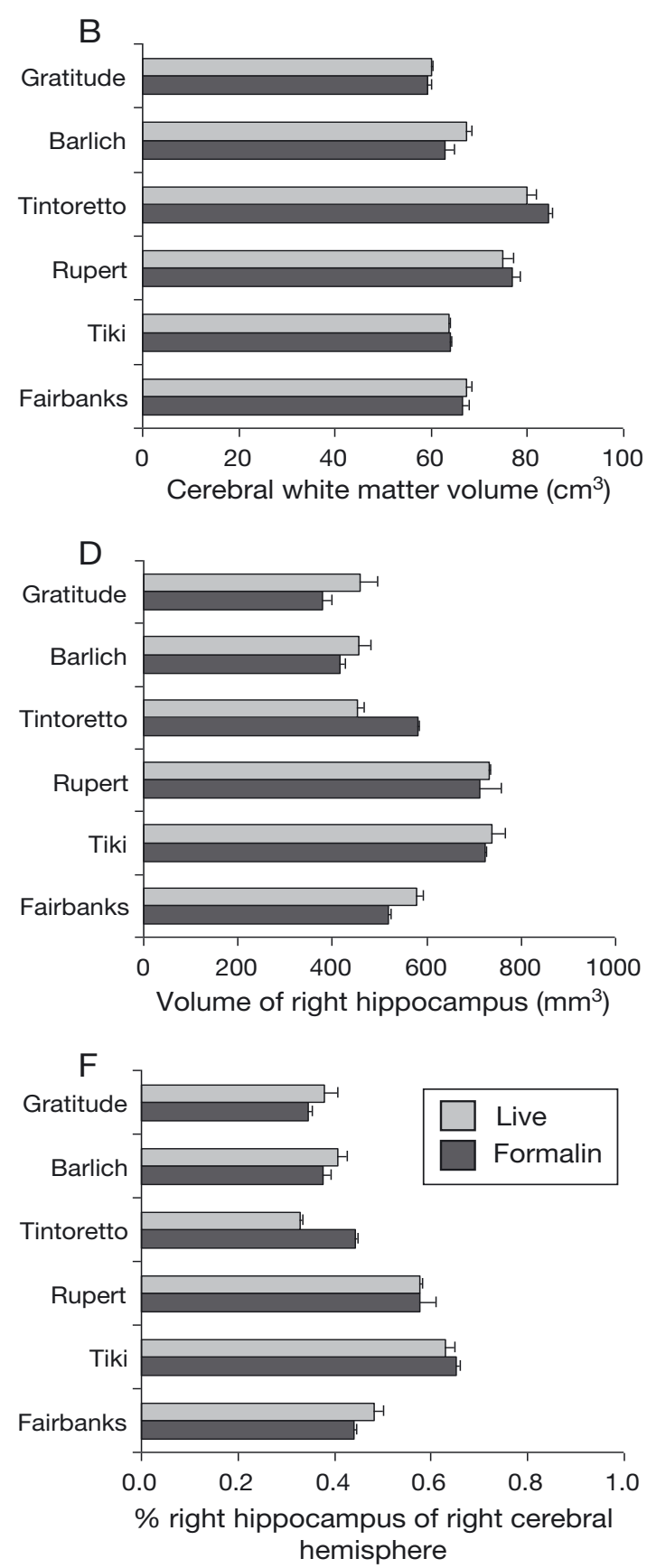

Fig. 5. Zalophus californianus. Volumes $\left(\mathrm{mm}^{3}\right.$ or $\left.\mathrm{cm}^{3}\right)$ of brain structures from live and formalin-fixed brains of 6 California sea lions. (A) Cerebral gray matter volume. (B) Cerebral white matter volume. (C,D) Volumes of (C) left and (D) right hippocampus. (E,F) Percent of the cerebral hemisphere occupied by (E) the left and (F) the right hippocampus. Fixation of the brain occurred at $2 \mathrm{~h}$ after death for 'Fairbanks', $3 \mathrm{~h}$ after death for 'Tiki', $4 \mathrm{~h}$ after death for 'Rupert', $23 \mathrm{~h}$ after death for 'Tintoretto', $48 \mathrm{~h}$ after death for 'Barlich' and $51 \mathrm{~h}$ after death for 'Gratitude'. Structural volumes were determined twice, which was the source of the error bars 
$48 \mathrm{~h}$ after death) provided an accurate method for detecting unilateral and bilateral atrophy that was comparable with live imaging. Imaging postmortemfixed brains provided some information on brain structure; however, the images of the hippocampus and surrounding structures were of poorer quality than images acquired from live animals and postmortemintact. Despite these problems, volumes of cerebral GM and WM, as well as hippocampus, were similar to volumes calculated from images acquired alive and were sufficient to detect hippocampal atrophy.

Thus, postmortem MRI scanning (either intact or formalin-fixed) with volumetric analysis can be used to investigate the acute, chronic and possible developmental effects of DA on the brain of California sea lions. While MRI will never replace histological evaluation, it does permit broad coverage of the brain with very thin sections, which can be helpful in localizing lesions. In addition, volumetric calculations of brain structures add a powerful quantitative dimension. These advantages can increase the chances of a definitive quantitative diagnosis. This approach can also be used to investigate the effects of other biological, chemical and physical agents on wild California sea lions, other marine mammals and wildlife in general.

Acknowledgements. The authors thank all the staff and volunteers at The Marine Mammal Center. They also thank Drs. $H$. Harris and F. Nutter for their veterinary assistance and Dr. G. Schneider for his expertise in neurobiology. The authors also thank J. Bloom and Dr. C. Kruse-Elliot for MRI and the IAMs imaging center, Redwood City, California for their assistance. This study was supported under a Subaward with the University Corporation of Atmospheric Research (UCAR) under Grant No. NA06OAR4310119 (Training Tomorrow's Ecosystem and Public Health Leaders Using Marine Mammals as Sentinels of Oceanic Change) with the National Oceanic and Atmospheric Administration (NOAA), US Department of Commerce. We also acknowledge the partners of this training grant: University of California Davis Wildlife Health Center, The Marine Mammal Center and Northwest Fisheries Science Center (NWFSC). Supplemental funding was provided by The Marine Mammal Center, the US National Marine Fisheries Service and Dr. D. Mann, College of Marine Science, University of South Florida.

\section{LITERATURE CITED}

> Colombo N, Berry I, Kucharczyk J, Kucharczyk W and others (1987) Posterior pituitary glands appearance on MR images in normal and pathologic state. Radiology 165: 481-485

Conrad PA, Miller MA, Kreuder C, James ER and others (2005) Transmission of toxoplasma: clues from the study of sea otters as sentinels of Toxoplasma gondii flow into the marine environment. Int J Parasitol 35:1155-1168

Costa LG, Giordano G (2007) Developmental neurotoxicity of polybrominated diphenyl ether (PBDE) flame retardants. Neurotoxicology 28:1047-1067
Dua-Sharma S, Sharma KN, Jacobs HL (1970) The canine brain in stereotaxic coordinates. MIT Press, Cambridge, MA

Feinberg DA, Mark AS (1987) Human brain motion and cerebrospinal fluid circulation demonstrated with MR velocity imaging. Radiology 163:793-799

Goldstein T, Mazet JAK, Zabka TS, Langlois G and others (2008) Novel symptomatology and changing epidemiology of domoic acid toxicosis in California sea lions (Zalophus californianus): an increasing risk to marine mammal health. Proc Biol Sci 275:267-276

Greig DJ, Gulland FMD, Kreuder C (2005) A decade of live California sea lion (Zalophus californianus) strandings along the Central California coast: causes and trends, 1991-2000. Aquat Mamm 31:11-22

ICVGAN (International Committee on Veterinary Gross Anatomical Nomenclature) (2005) Nomina anatomica veterinaria, 5th edn. World Association of Veterinary Anatomists, Ithaca, NY

Jepson PD, Arbelo M, Deaville R, Patterson IAP and others (2003) Gas-bubble lesions in stranded cetaceans. Nature 425:575-576

Keller SS, Roberts N (2008) Voxel-based morphometry of temporal lobe epilepsy: an introduction and review of the literature. Epilepsia 49:741-757

Lisanti C, Carlin C, Banks KP, Wang D (2007) Normal MRI appearance and motion-related phenomena of CSF. Am J Roentgenol 188:716-725

Malko JA, Hoffman JC, McClees EC, Davis PC, Braun IF (1988) A phantom study of intracranial CSF signal loss due to pulsatile motion. Am J Neuroradiol 9:83-89

> Marino L, Murphy TL, Deweerd AL, Morris JA and others (2001a) Anatomy and three-dimensional reconstructions of the brain of the white whale (Delphinapterus leucas) from magnetic resonance images. Anat Rec 262:429-439

> Marino L, Murphy TL, Gozal L, Johnson JI (2001b) Magnetic resonance imaging and three dimensional reconstructions of the brain of a fetal common dolphin, Delphinus delphis. Anat Embryol 203:393-402

Marino L, Sudheimer KD, Murphy TL, Davis KK and others (2001c) Anatomy and three-dimensional reconstructions of the brain of a bottlenose dolphin (Tursiops truncatus) from magnetic resonance images. Anat Rec 264:397-414

Marino L, Sudheimer K, Sarko D, Sirpenski G, Johnson JI (2003a) Neuroanatomy of the harbor porpoise (Phocoena phocoena) from magnetic resonance images. J Morphol 257:308-347

> Marino L, Sudheimer K, Pabst DA, McLellan WA, Johnson JI (2003b) Magnetic resonance images of the brain of a dwarf sperm whale (Kogia simus). J Anat 203:57-76

> Marino L, Sudheimer K, McLellan WA, Johnson JI (2004a) Neuroanatomical structures of the spinner dolphin (Stenella longirostris orientalis) brain from magnetic resonance images. Anat Rec 279A:601-610

> Marino L, Sherwood CC, Delman BN, Tang CY, Naidich TP, Hof PR (2004b) Neuroanatomy of the killer whale (Orcinus orca) from magnetic resonance images. Anat Rec 281A: $1256-1263$

> Montie EW, Schneider GE, Ketten DR, Marino L, Touhey KE, Hahn ME (2007) Neuroanatomy of the subadult and fetal brain of the Atlantic white-sided dolphin (Lagenorhynchus acutus) from in situ magnetic resonance images. Anat Rec 290:1459-1479

> Montie EW, Schneider GE, Ketten DR, Marino L, Touhey KE, Hahn ME (2008) Volumetric neuroimaging of the brain of the Atlantic white-sided dolphin (Lagenorhynchus acutus). Anat Rec 291:263-282 
Montie EW, Pussini N, Schneider GE, Battey TWK, Dennison S, Barakos J, Gulland F (2009) Neuroanatomy and volumes of brain structures of a live California sea lion (Zalophus californianus) from magnetic resonance images. Anat Rec 292:1523-1547

Nolte J, Angevine JB (2000) The human brain in photographs and diagrams, 2nd edn. Mosby, St. Louis, MO

Ramsdell JS, Zabka TS (2008) In utero domoic acid toxicity: a fetal basis to adult disease in the California sea lion (Zalophus californianus). Mar Drugs 6:262-290

Scholin CA, Gulland F, Doucette GJ, Benson S and others (2000) Mortality of sea lions along the central California coast linked to a toxic diatom bloom. Nature 403:80-84

Sharlin DS, Bansal R, Zoeller RT (2006) Polychlorinated

Editorial responsibility: Michael Moore,

Woods Hole, Massachusetts, USA biphenyls exert selective effects on cellular composition of white matter in a manner inconsistent with thyroid hormone insufficiency. Endocrinology 147:846-858

Silvagni PA, Lowenstine LJ, Spraker T, Lipscomb TP, Gulland FMD (2005) Pathology of domoic acid toxicity in California sea lions (Zalophus californianus). Vet Pathol 42:184-191

Tiedeken JA, Ramsdell JS (2010) Zebrafish seizure model identifies $p, p^{\prime}$ - DDE as the dominant contaminant of fetal California sea lions that accounts for synergistic activity with domoic acid. Environ Health Perspect 118:545-551

Zoeller RT, Dowling ALS, Herzig CTA, Iannacone EA, Gauger KJ, Bansal R (2002) Thyroid hormone, brain development, and the environment. Environ Health Perspect 110:355-361

Submitted: April 26, 2010; Accepted: June 29, 2010

Proofs received from author(s): August 30, 2010 